Mrtchell, P. \& Moyle, J. (1956). J. gen. Microbiol. 15, 512-520

\title{
Liberation and Osmotic Properties of the Protoplasts of Micrococcus lysodeikticus and Sarcina lutea
}

\author{
BY P. MITCHELL AND JENNIFER MOYLE \\ Zoology Department, University of Edinburgh
}

SUMMARY: Stable protoplasts may be released from Micrococcus lysodeikticus and Sarcina lutea by digestion of the cell-wall with lysozyme in sucrose or $\mathrm{NaCl}$ solutions having an osmotic pressure of some 25 atmospheres, but not in glycerol solutions of the same osmotic pressure. The stability of the protoplasts depends not only upon the depression of the water activity by the solute but upon an osmotic pressure exerted against the protoplast (plasma-) membrane. The permeability of the protoplast membrane to a number of solutes resembles that of the osmotic barrier of intact Staphylococcus aureus.

This paper describes work on the protoplasts of Micrococcus lysodeikticus and Sarcina lutea which was completed just over a year ago in the Department of Biochemistry, University of Cambridge. It was undertaken as part of a research programme designed to investigate the physiological role of the plasma-membrane in bacteria (Mitchell \& Moyle, 1956a,b).

Three years ago, Weibull (1953) discovered that spherical bodies, which were thought to be intact protoplasts, emerged from Bacillus megaterium when the cell walls were digested by lysozyme in solutions of which the water activity had been depressed by the addition of polyethylene glycol, sucrose or certain other solutes. It was not known, however, whether the mechanism of the stabilizing action of the solutes was by the exertion of an osmotic pressure across the membrane, thus preventing osmotic explosion of the protoplast, or whether the solutes prevented the swelling of hydrated constituents of the protoplasm which would otherwise have ruptured the external membrane. It was therefore of interest to measure the permeability of isolated protoplasts to a range of solutes; for it would be expected that if the protoplasts were stabilized by the external osmotic pressure, only non-penetrating solutes would be effective as stabilizers, whereas if the mechanism of stabilization were by dehydration of components of the cytoplasm the effectiveness of a solute would depend only upon the lowering of the activity of the water. The lysozyme-sensitive cocci were chosen for study because we wished to obtain data which might usefully be compared with similar data on the internal osmotic pressure and permeability of Staphylococcus aureus.

Grula \& Hartsell (1954) attempted to prepare protoplasts of Micrococcus lysodeikticus by the method used for Bacillus megaterium (Weibull, 1953), but most of the protoplasts swelled and disintegrated as soon as they were released from the cell-walls. Dr M. R. J. Salton (personal communication) attempted to obtain protoplasts from $\boldsymbol{M}$. lysodeikticus in higher sucrose concentrations, but could observe no indication of protoplast formation from the changes in light- 
scattering of the suspensions in sucrose. In view of our observations on Staphylococcus aureus which implied that the osmotic pressure was between 20 and 25 atmospheres (Mitchell \& Moyle, 1956a), it was possible that the protoplasts of the cocci might require at least $\mathrm{M}$-sucrose or $\mathrm{M}$-saline solutions to preserve them from osmotic explosion. Moreover, it seemed possible that at very high sucrose concentrations, the depression of the light scattering which would result from the elevation of the refractive index of the medium might be sufficient to make the protoplasts invisible by light scattering unless they were subsequently transferred to a medium of lower refractive index.

\section{METHODS}

Growth and preparation of organisms. Cultures of Micrococcus lysodeikticus (NCTC 2665) and Sarcina lutea (laboratory strain) were grown aerobically at $25^{\circ}$ in a medium containing $3 \%(\mathrm{w} / \mathrm{v})$ tryptic digest of casein, $1 \%$ glucose and $0 \cdot 1 \%$ Marmite, using the rotated flask technique (Mitchell, 1949). The organisms were harvested at a concentration equivalent to 1.5-2 mg. dry weight/ml., washed twice with distilled water (for $M$. lysodeikticus) or $0.5 \mathrm{M}-\mathrm{NaCl}$ (for $S$. lutea) and suspended at a concentration corresponding to $c .100 \mathrm{mg}$. dry weight $/ \mathrm{ml}$. in distilled water (for M. lysodeikticus) or $0.5 \mathrm{M}-\mathrm{NaCl}$ (for S. lutea).

Measurement of turbidity. The extinction of $1 \mathrm{~cm}$. depth of suspension was measured at a wavelength of $700 \mathrm{~m} \mu$., using the Beckman model DU spectrophotometer.

Microscopy. The intact organisms and protoplasts were examined with a Cooke Troughton and Simms binocular phase-contrast microscope in films of aqueous solution sealed between slide and coverslip with vaseline.

\section{Protoplast release and stability}

Method I. Visibility of protoplasts and intact organisms in $\mathrm{NaCl}$ and sucrose solutions. Samples of organisms were incubated for $2 \mathrm{hr}$. at $25^{\circ}$ at a standard concentration corresponding to $200 \mu \mathrm{g}$. dry wt. organisms $/ \mathrm{ml}$., with a range of concentrations of $\mathrm{NaCl}$ or sucrose in $0.04 \mathrm{M}$-sodium phosphate buffer (pH 6.8), containing $10 \mu \mathrm{g}$. (for Micrococcus lysodeiktïcus) or $40 \mu \mathrm{g}$. (for Sarcina lutea) crystalline egg-white lysozyme/ml. The turbidities of the suspensions were then measured and compared with those of controls in which the lysozyme was omitted.

Method II. Dependence of protoplast stability on osmotic pressure of releasemedium. Samples of suspensions were incubated as above but at a concentration corresponding to $10 \mathrm{mg}$. dry wt. organisms $/ \mathrm{ml}$. Samples $(0.1 \mathrm{ml}$.) of the incubated suspensions were pipetted into $5 \mathrm{ml}$. samples of $\mathrm{M}-\mathrm{NaCl}$ containing 0.01 M-sodium phosphate buffer at $\mathrm{pH} \mathrm{6.8}$. After $20 \mathrm{~min}$. at $20^{\circ}$, the turbidities of the suspensions were measured. Controls were done omitting lysozyme only.

Method III. Dependence of protoplast stability on osmotic pressure of $\mathrm{NaCl}$ solutions to which protoplasts are transferred after release in ' $\mathrm{NaCl}$ or sucrose. The protoplasts were released by incubating the organisms with lysozyme at $25^{\circ}$ for $2 \mathrm{hr}$. at a concentration corresponding to $10 \mathrm{mg}$. dry wt. organisms $/ \mathrm{ml}$. 
in 1.2 $\mathrm{M}$-sucrose or $2 \mathrm{~m}-\mathrm{NaCl}$ containing phosphate buffer as in (I). Samples $(0.1 \mathrm{ml}$.) of the protoplast suspension were pipetted into $5 \mathrm{ml}$. samples of $\mathrm{NaCl}$ ranging from 0 to $2 \mathrm{M}$, containing $0.01 \mathrm{~m}$-sodium phosphate buffer at $\mathrm{pH} 6.8$. After $20 \mathrm{~min}$. the turbidities of the suspensions were measured. Controls were done omitting lysozyme only.

Method IV. Dependence of protoplast stability on osmotic pressure of sucrose, $\mathrm{NaCl}$ or glycerol solutions to which protoplasts are transferred after release in $1 \cdot 2$ M-sucrose. The protoplasts were released in $1.2 \mathrm{M}$-sucrose as in method III. The incubated suspension was mixed with 5 vol. $1 \cdot 2 \mathrm{M}-\mathrm{NaCl}$ (to decrease the specific gravity) and centrifuged at $20^{\circ}$; and the protoplasts were redispersed in $1.2 \mathrm{M}-\mathrm{NaCl}$ to give a suspension density corresponding to $100 \mathrm{mg}$. original dry wt. organisms $/ \mathrm{ml}$. Samples $(0.5 \mathrm{ml}$.) of the protoplast suspension were pipetted into $4.5 \mathrm{ml}$. samples of $\mathrm{NaCl}$, sucrose or glycerol arranged to give final concentrations ranging from 0 to $2 \mathrm{M}$ in $0.01 \mathrm{M}$-sodium phosphate buffer at pH 6.8. After $30 \mathrm{~min}$. incubation at $25^{\circ}$, samples $(0 \cdot 1 \mathrm{ml}$.) of the protoplast suspensions in the $\mathrm{NaCl}$, sucrose and glycerol solutions were pipetted into $5 \mathrm{ml}$. samples of $1.2 \mathrm{M}-\mathrm{NaCl}$ containing $0.01 \mathrm{M}$-sodium phosphate buffer at pH 6.8, and after a further 20 min. the turbidities were measured.

Method V. Determination of protoplast permeability. The protoplasts were released in sucrose as in method III. The incubated suspension was mixed with $5 \mathrm{vol} .1 \cdot 2 \mathrm{M}-\mathrm{NaCl}$ and centrifuged at $20^{\circ}$; and the protoplasts were redispersed in $1.2 \mathrm{M}-\mathrm{NaCl}$ to give a suspension density corresponding to $10 \mathrm{mg}$. original dry weight organisms $/ \mathrm{ml}$. Samples $(0.1 \mathrm{ml}$.) of the protoplast suspension were pipetted into 1.5 molal solutions (in $0.01 \mathrm{M}$-sodium phosphate buffer at $\mathrm{pH} \mathrm{6.8}$ ) of the solutes to which the permeability was to be measured. The rate of lysis of the protoplasts, measured by the change of light scattering, was taken as an approximate index of the rate of permeation of the solute.

\section{RESULTS}

'Protoplast' release

The effect of lysozyme treatment (Method I) on the turbidity of suspensions of Micrococcus lysodeikticus in concentrations of sucrose from 0.1 to $1.0 \mathrm{M}$ is shown in Fig. 1. At the low sucrose concentrations the lysozyme-treated suspensions scattered very little light, but, although the amount of light scattered was still small, at concentrations of sucrose between 0.5 and $1.0 \mathrm{M}$ they scattered between 10 and $20 \%$ as much light as the corresponding untreated suspensions. This suggests that protoplasts were being liberated and were stable in sucrose concentrations approaching $1 \mathrm{~m}$, but that their light scattering (like that of the intact organisms) was depressed by the high refractive index of the sucrose solutions. The lysozyme treatment was therefore done in $\mathrm{NaCl}$ solutions (method $\mathrm{I}$ ) with the results shown in Fig. 2. At concentrations of $\mathrm{NaCl}$ above $0.5 \mathrm{M}$ the lysozyme-treated organisms scattered between 50 and $60 \%$ as much light as the untreated organisms, but below $0.5 \mathrm{M}-\mathrm{NaCl}$ the light scattered by the lysozyme-treated organisms fell towards some $5 \%$ of that scattered by the untreated organisms. Similar results were 


\section{Protoplasts of cocci}

obtained with Sarcina lutea, the only significant difference being that $S$. lutea tended to lyse slowly in distilled water. The suspensions of $S$. lutea were therefore washed and made up in $0.5 \mathrm{M}-\mathrm{NaCl}$ in place of distilled water.

Phase-contrast microscopy showed that the lysozyme-treated suspensions of both organisms in concentrations of $\mathrm{NaCl}$ above $1.0 \mathrm{M}$ contained spherical bodies of approximately the same diameter but of lower contrast than organisms of corresponding control suspensions, and whereas the organisms of the controls adhered to one another the spherical bodies in the lysozymetreated suspensions were separate. In concentrations of $\mathrm{NaCl}$ below $1.0 \mathrm{M}$, the lysozyme-treated suspensions contained spherical bodies (which were larger than the cells of corresponding control suspensions) together with ghost membranes of extremely low contrast. The lower the $\mathrm{NaCl}$ concentration, the larger

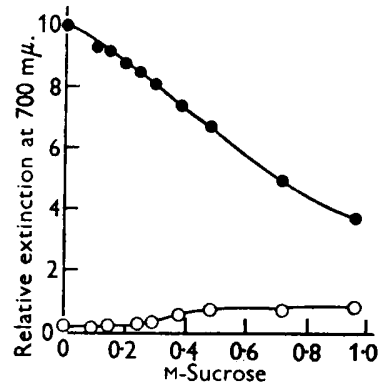

Fig. 1

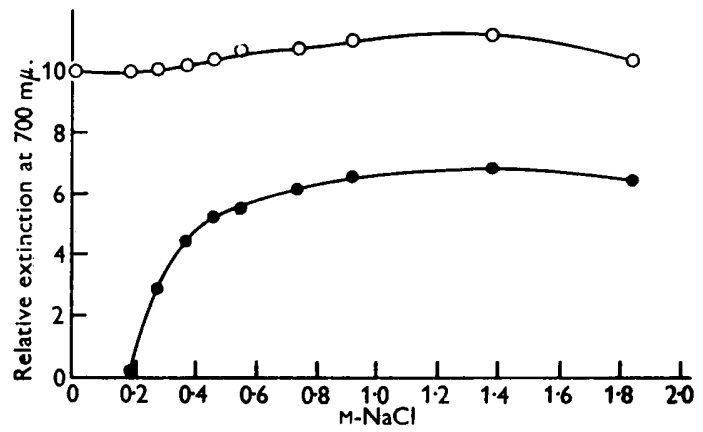

Fig. 2

Fig. 1. The dependence of the extinction of suspensions of Micrococcus lysodeikticus on the concentration of sucrose in the suspension medium after incubation with lysozyme $(O)$ and without lysozyme (O) (see method I).

Fig. 2. The dependence of the extinction of suspensions of Micrococcus lysodeikticus on the concentration of $\mathrm{NaCl}$ in the suspension medium after incubation with lysozyme (O) and without lysozyme $(O)$ (see method I).

was the ratio of ghosts to intact spherical bodies; and no intermediate forms were present. It was evident that the spherical bodies corresponded to the lightscattering units of the suspensions and that since they either remained intact or lysed completely, the amount of light scattered by a suspension was approximately proportional to the number of surviving spherical bodies. Microscopic examination of the suspensions treated with lysozyme in sucrose (corresponding to Fig. 1) was not successful because the contrast was much decreased by the high refractive index of the sucrose solutions; but by diluting the sucrose-containing suspensions with $\mathrm{M}-\mathrm{NaCl}$, the same general pattern as described above could be observed.

In order to ascertain quantitatively whether lysozyme treatment in $\mathrm{NaCl}$ solutions followed the same pattern as in sucrose solutions, samples of organisms were treated with lysozyme in a range of sucrose and $\mathrm{NaCl}$ concentrations, and subsequently transferred to $\mathrm{M}-\mathrm{NaCl}$ to measure the turbidity (method II). Fig. 3 shows the results of such an experiment with Micrococcus lysodeikticus, harvested at a population density corresponding to $c .1 .5 \mathrm{mg}$. dry weight $/ \mathrm{ml}$.; 
similar results were obtained with Sarcina lutea. It is evident that to stabilize $50 \%$ of the light-scattering units of the lysozyme-treated suspension requires the presence of $0.83 \mathrm{M}-\mathrm{NaCl}$ or $0.53 \mathrm{M}$-sucrose. From the vapour-pressure equilibrium data of Robinson \& Sinclair (1934), it is found that 0.53 M-sucrose corresponds to $0.34 \mathrm{M}-\mathrm{NaCl}$ in water activity (or osmotic pressure for a membrane impermeable to sucrose and $\mathrm{NaCl}$ ). Thus, the stabilization of the lysozyme-treated suspensions is practically the same whether the water activity is depressed (or osmotic pressure raised) by $\mathrm{NaCl}$ or by sucrose.

The stabilization of the lysozyme-treated organisms in the higher $\mathrm{NaCl}$ and sucrose concentrations might have been due either to stabilization of protoplasts which had been completely released from the cell walls, or to an inhibitory effect of the sucrose and $\mathrm{NaCl}$ on the lysozyme reaction. The latter

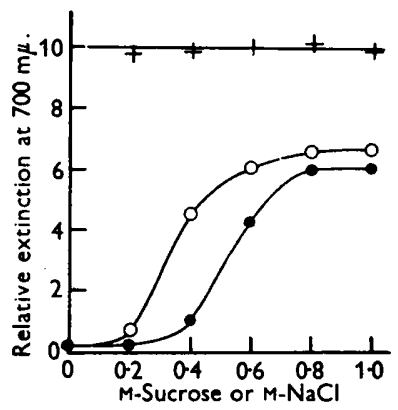

Fig. 3

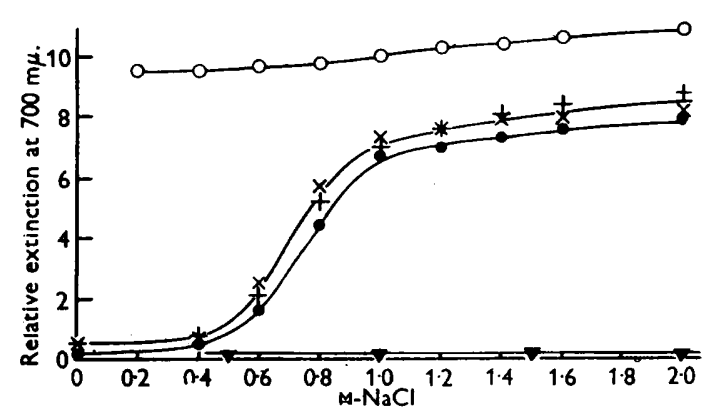

Fig. 4

Fig. 3. The dependence of the extinction of suspensions of Micrococcus lysodeikticus in $\mathrm{M}-\mathrm{NaCl}$ on the concentration of $\mathrm{NaCl}$ or sucrose in which the cells were previously incubated with lysozyme $(\mathrm{NaCl}, \mathrm{O}$; sucrose, $O$ ) and without lysozyme (mean for $\mathrm{NaCl}$ and sucrose + ) (see method II).

Fig. 4. The dependence of the extinction of suspensions of Sarcina lutea on the concentration of $\mathrm{NaCl}$ to which the cells are transferred after incubation in $2 \mathrm{M}$-glycerol, $1.2 \mathrm{M}$-sucrose, or $\mathrm{M}$ or $2 \mathrm{M}-\mathrm{NaCl}$ solutions with lysozyme (2M-glycerol, $\nabla ; 1 \cdot 2 \mathrm{M}$-sucrose, $\bigcirc$; $\mathrm{M}-\mathrm{NaCl}, \times$; $2 \mathrm{M}-\mathrm{NaCl},+$ ) and without lysozyme (mean for $\mathrm{NaCl}$, sucrose and glycerol, $\mathrm{O}$ ) (see method III).

possibility was eliminated by showing that the organisms in the suspensions treated with lysozyme at high sucrose or $\mathrm{NaCl}$ concentrations lysed when transferred to solutions of lower concentration. The organisms were treated with lysozyme in $1.2 \mathrm{M}$-sucrose, or $\mathrm{M}$ or $2 \mathrm{M}-\mathrm{NaCl}$ solutions and subsequently transferred to a range of $\mathrm{NaCl}$ concentrations (method III). Fig. 4 shows such an experiment with Sarcina lutea. The turbidity of the suspensions that were treated with lysozyme in $\mathrm{m}$ or $2 \mathrm{M}-\mathrm{NaCl}$ was slightly higher than those treated with lysozyme in $1.2 \mathrm{M}$-sucrose over the whole range of $\mathrm{NaCl}$ concentrations to which they were transferred. However, the dependence of the stability of the light-scattering elements upon the $\mathrm{NaCl}$ concentration to which the suspensions were transferred was practically the same whether lysozyme treatment was done in $1.2 \mathrm{M}$-sucrose, $\mathrm{M}$ or $2 \mathrm{M}-\mathrm{NaCl}$. It followed that the action of the high concentrations of sucrose or $\mathrm{NaCl}$ was not due to an inhibition of the lysozyme reaction, but could be attributed to stabilization of 
'protoplasts' which had been completely released from the normal mechanical protection of the cell wall.

In order to determine whether the 'protoplasts' were stabilized by an osmotic pressure acting across a semi-permeable membrane, or whether the stabilization was due to dehydration of protoplasmic constituents which would otherwise swell and cause the 'protoplasts' to disintegrate, the lysozyme treatment was carried out in $2 \mathrm{M}$-glycerol in place of sucrose (method III); for it was shown by Fischer(1903) that many bacteria are permeable to glycerol. The results (Fig. 4), showed that the 'protoplasts' were not stabilized when released in $2 \mathrm{M}$-glycerol. The stabilization cannot therefore be due to lowering of the water activity alone: it must be caused by the substitution of an osmotic

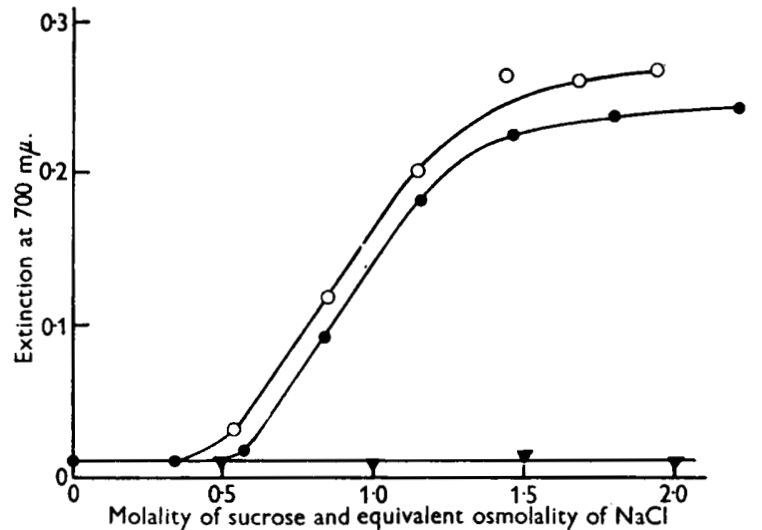

Fig. 5

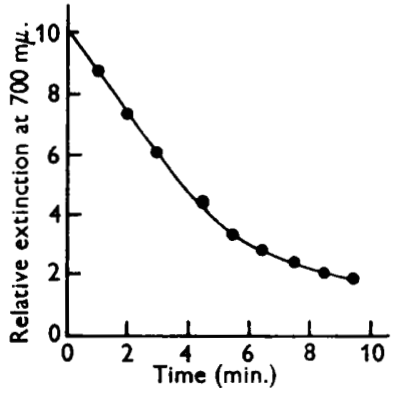

Fig. 6

Fig. 5. The dependence of the extinction of suspensions of 'protoplasts' of Micrococcus lysodeikticus in $1.2 \mathrm{M}-\mathrm{NaCl}$ on the concentration of glycerol $(\nabla)$, suerose $(O)$ or $\mathrm{NaCl}(O)$ to which they are intermediately transferred after release by lysozyme in $1.2 \mathrm{M}$-sucrose (see method IV).

Fig. 6. The dependence of the extinction of 'protoplasts' of Micrococcus lysodeikticus suspended in $1.5 \mathrm{molal} \mathrm{D}$-ribose on time at $20^{\circ}$ (see method $\mathrm{V}$ ).

pressure at the outer surface of the 'protoplast' membrane for the hydrostatic pressure exerted by the cell-wall of the intact organism. The molarity of $\mathrm{NaCl}$ at which half the 'protoplasts' of Sarcina lutea are stabilized is $c .0 .7 \mathrm{~m}$ in the experiment of Fig. 4: it corresponds to an osmotic pressure of between 25 and 30 atmospheres. This probably represents about the mean osmotic pressure of the cells.

An alternative and perhaps preferable method of examining the dependence of the stability of the 'protoplasts' on the osmotic pressure and composition of the suspension medium is to release a large batch of 'protoplasts' in $1.2 \mathrm{M}$-sucrose, to transfer samples of these 'protoplasts' to ranges of concentration of $\mathrm{NaCl}$, sucrose and glycerol, and then after a fixed time interval to determine the proportion of surviving 'protoplasts' by measuring their light-scattering when transferred to $1.2 \mathrm{M}-\mathrm{NaCl}$ (method IV). The results of such an experiment with Micrococcus lysodeikticus are plotted in Fig. 5. The scale of concentration has been converted to molality of sucrose and equivalent osmolality of $\mathrm{NaCl}$ by 
means of the vapour pressure equilibrium data of Robinson \& Sinclair (1934) and specific gravity tables. As with Sarcina lutea, sucrose and $\mathrm{NaCl}$ are about equally effective in stabilizing the 'protoplasts' from $\boldsymbol{M}$. lysodeikticus while glycerol is not effective. The osmolality required to stabilize half the "protoplasts' is c. $0 \cdot 9$, corresponding to an osmotic pressure of $c .20$ atmospheres. The osmolality of sucrose or $\mathrm{NaCl}$ solutions required to stabilize half the "protoplasts' of $\boldsymbol{M}$. lysodeikticus and $\boldsymbol{S}$. lutea varied from one culture to another about a mean of 1.0 osmolal, when harvested at a dry weight of $2.0 \mathrm{mg} . / \mathrm{ml}$.

\section{'Protoplast' permeability}

The rate of lysis of the 'protoplasts' of Micrbcoccus lysodeikticus and Sarcina lutea was less than $10 \% / \mathrm{hr}$. at $20^{\circ}$ in 1.5 molal solutions of the following solutes in 0.01 M-sodium phosphate buffer at pH 6.8 (except where otherwise stated): $\mathrm{NaCl}, \mathrm{KCl}, \mathrm{NH}_{4} \mathrm{Cl}, \mathrm{MgCl}_{2}, \mathrm{NaBr}, \mathrm{KBr}, \mathrm{Na}$-acetate (pH 9), $\mathrm{K}$-acetate ( $\mathrm{pH} \mathrm{9}), \mathrm{K}_{2} \mathrm{SO}_{4},\left(\mathrm{KH}_{2} \mathrm{PO}_{4}+\mathrm{K}_{2} \mathrm{HPO}_{4}\right)$, Na-glutamate, lysine-HCl, D-glucose, D-fructose, D-mannose, D-galactose, D-sorbose and sucrose (method V). Since it was possible that metabolism of the carbohydrates might affect the stability of the 'protoplasts', the effect of the presence of $10^{-2} \mathrm{M}-\mathrm{KCN}, 10^{-2} \mathrm{M}$-sodium iodoacetate, $10^{-3} \mathrm{M}$-sodium dinitrophenate or $10^{-5} \mathrm{M}-\mathrm{HgCl}_{2}$ on the rate of lysis of the 'protoplasts' was determined. The rate of lysis was, as in the case of the untreated suspensions, less than $10 \% / \mathrm{hr}$. The 'protoplast' membrane is therefore only slightly permeable to the above solutes under the conditions of these experiments. However, under the same conditions as above, the 'protoplasts' were $c .70 \%$ lysed in 3 sec. in glycerol, 20 sec. in erythritol, $5 \mathrm{~min}$. in D-ribose, $30 \mathrm{~min}$. in L-arabinose and $1 \mathrm{hr}$. in D-sorbitol. The progress of lysis of 'protoplasts' of Micrococcus lysodeikticus in D-ribose is shown in Fig. 6.

It is not possible to estimate accurately the rate of equilibration of the solutes across the 'protoplast' membrane from measurements such as those of Fig. 6 without much additional information. However, an approximate estimate of the permeability of the membrane can be obtained since we know from the data of Figs. 4 and 5 that $70 \%$ lysis of the 'protoplasts' corresponds to about an osmotic pressure difference of 0.75 osmolar across the 'protoplast' membrane, or to half equilibration of the $1.5 \mathrm{molal}$ non-electrolyte solutions used in these experiments. Thus, we may say that the $70 \%$ lysis times given above correspond approximately to the times for half equilibration of the solutes across the 'protoplast' membrane.

\section{DISCUSSION}

There is no doubt that the 'protoplasts' liberated from Micrococcus lysodeikticus and Sarcina lutea by lysozyme possess the properties that would be expected of the protoplasm of the cell covered by a plasma membrane. It is, however, important to emphasize that we do not yet know whether the composition of the membrane of the 'protoplasts' liberated by lysozyme is the 


\section{Protoplasts of cocci}

same as that of the plasma-membrane of the intact organism. Even so, we suggest that the removal of the inverted commas from the word protoplast when used to describe the bodies which are liberated from $\boldsymbol{M}$. lysodeikticus and $S$. lutea by lysozyme in media of high osmotic pressure is justified on the following grounds: (i) Lysozyme is known to dissolve the cell wall of these organisms (Salton, 1954). (ii) The bodies are approximately the same size as the intact organisms, and of only slightly lower contrast in phase-contrast microscopy, when suspended in media in which the degree of lysis is small. (iii) The bodies scatter about two-thirds as much light as the intact organisms. (iv) The bodies are covered by a semipermeable membrane which retains the small molecular weight internal solutes of which the total osmotic pressure is some 20 atmospheres, and the bodies are osmotically exploded in media of low osmotic pressure. (v) After lysis in media of low osmotic pressure the empty membranes are visible by phase-contrast microscopy. (vi) In media containing a high concentration of solutes known to be unable to penetrate the plasma membrane of Staphylococcus aureus and Escherichia coli (Mitchell \& Moyle, $1956 a$ ), the membrane of the bodies is subject to an external osmotic pressure which substitutes for the hydrostatic pressure normally exerted by the cell wall and prevents explosion of the bodies. (vii) When the osmotic pressure of the external medium is raised by glycerol or other solutes which are known to penetrate the plasma membrane of intact bacteria, the passage of the solute through the 'protoplast' membrane results in osmotic explosion.

Since this work was completed, Weibull (1955) has described experiments on the osmotic properties of the protoplasts of Bacillus megaterium with which our observations are essentially in agreement. He observed, however, that the total volume of the protoplasts of $\boldsymbol{B}$. megaterium was independent of the osmotic pressure of the sucrose solution in which they were released, over a range from $0 \cdot 125$ to $0 \cdot 4 \mathrm{M}$. In the work described here, there was no indication that the protoplasts of the cocci distinguished between the osmotic pressure of the medium in which they were released and that to which they were subsequently transferred. It may, perhaps, be relevant that whereas the protoplasts of $B$. megaterium must change shape from cylinders to spheres, there is little or no change of shape of the protoplasts of the cocci during release by lysozyme.

The aim of the work described in the present paper was to obtain a general view of the effectiveness of the membrane of Micrococcus lysodeikticus and Sarcina lutea as a barrier to the free diffusion of solutes between the cell interior and the environment. It is, perhaps, relevant to point out that the membrane does not act only as an osmotic barrier. Its most specific function is to link the internal and external media, allowing entry of specific metabolites and exit of end-products of metabolism. The rate of lysis of the protoplasts in 1.5 molal glucose and in the other hexose solutions shows that these sugars enter both the normal (semi-anaerobic) and the inhibitor-treated protoplasts at a rate of less than 0.1 mole/l. protoplast volume/hr. On the other hand, we have recently found that under aerobic conditions the intact cocci or the protoplasts oxidize glucose at a rate corresponding to 1.0 mole or more/l. 
protoplast volume/hr. It follows that the movement of glucose through the plasma membrane is closely linked to its oxidation. The implication of this coupling has been discussed briefly elsewhere (Mitchell \& Moyle, 1956 $b$ ), and we have suggested that glucose is carried through the membrane on an enzyme or on a protein carrier of equivalent specificity and affinity. Further work on this problem, which is now in progress, will be described shortly in another paper.

We are indebted to the Scottish Hospital Endowments Research Trust for the receipt of personal grants and to the Rockefeller Foundation for a grant for apparatus.

\section{REFERENCES}

Fischer, A. (1903). Vorlesungen über Bakterien, 2nd ed. Jena: Fischer.

Grula, E. A. \& Hartsell, S. E. (1954). Lysozyme and morphological alterations induced in Micrococcus lysodeikticus. J. Bact. 68, 171.

Mrtchell, P. (1949). A new technique for stirred aerated culture. Nature, Lond. $164,846$.

Mitchell, P. \& MoYle, J. (1956a). Osmotic function and structure in bacteria. In Bacterial Anatomy. Symp. Soc. gen. Microbiol. 6, 150.

Mitcheld, P. \& Moyle, J. (1956 b). Permeation mechanism in bacterial membranes. In Membrane Phenomena. Faraday Soc. General Discussion (in the Press).

Robinson, R. A. \& Sinclair, D. A. (1934). The activity coefficients of the alkali chlorides and of lithium iodide in aqueous solution from vapour pressure measurements. J. Amer. chem. Soc. 56, 1830.

Salton, M. R. J. (1954). The action of lysozyme on the cell walls of certain bacteria. J. gen. Microbiol. 11, ix.

Weibull, C. (1953). The isolation of protoplasts from Bacillus megaterium by controlled treatment with lysozyme. J. Bact. 66, 688.

WeIbULl, C. (1955). Osmotic properties of protoplasts of Bacillus megaterium. Exp. Cell Res. 9, 294. 